\title{
Fluorescence in situ hybridization testing of chromosomes 6, 8,9 and 11 in melanocytic tumors is difficult to automate and reveals tumor heterogeneity in melanomas
}

\author{
ARNAUD UGUEN $^{1-3}$, MARIE UGUEN $^{2}$, MATTHIEU TALAGAS $^{2-4}$, ERIC GOBIN $^{2}$, \\ PASCALE MARCORELLES ${ }^{2-4}$ and MARC DE BRAEKELEER ${ }^{1,3,5}$ \\ ${ }^{1}$ Inserm, U1078, F-29609 Brest; ${ }^{2}$ Department of Pathology, Brest University Hospital, \\ F-29220 Brest; ${ }^{3}$ European University of Brittany; ${ }^{4}$ EA4685, Brest Medical School; \\ ${ }^{5}$ Department of Cytogenetics and Reproduction Biology, Brest University Hospital, F-29220 Brest, France
}

Received November 22, 2015; Accepted June 16, 2016

DOI: 10.3892/ol.2016.4949

\begin{abstract}
Malignant melanomas may be difficult to differentiate from benign nevi on the basis of histology. Contrary to nevi, the majority of melanomas harbor chromosomal imbalances. Comparative genomic hybridization-based and fluorescence in situ hybridization (FISH) tests can help differentiating malignant from benign tumors. In the present study, eight-bacterial artificial chromosome (BAC) probes targeting chromosomes 6, 8, 9 and 11 were tested by FISH, and compared with a commercial four-color FISH probe set targeting chromosomes 6 and 11 in a first set of 62 tissue microarray-included melanocytic tumors (47 melanomas and 15 nevi). A second set of 108 tumors (70 melanomas and 38 nevi) was analyzed with the eight-probes kit, and manual counting was compared with the newly developed automated FISH signals counting and with semi-quantitative visual detection of chromosomal imbalances. Intra-tumor heterogeneity was also evaluated in 12 melanomas and 10 patients with paired melanoma samples. Testing the tumors from the first set with the commercial kit and the eight-probes test permitted to correctly identify 45/47 and 47/47 melanomas, respectively. In the second tumor set, $65 / 70$ malignant tumors presented at least one chromosomal imbalance, whereas none was detected in the nevi. The agreement between manual and automated signals counting was better in good-quality FISH slides compared with poor-quality slides. Semi-quantitative visual appreciation of chromosomal imbalances also reached strong agreement with exact manual counting. In addition, a frequent cytogenetic heterogeneity within melanomas and between paired tumors was noticed in patients with metastatic melanomas. To conclude, FISH testing
\end{abstract}

Correspondence to: Dr Arnaud Uguen, Department of Pathology, Brest University Hospital, 5 Avenue Foch, F-29220 Brest, France E-mail: arnaud.uguen@chu-brest.fr

Key words: melanoma, nevus, chromosome, fluorescence in situ hybridization, tumor heterogeneity targeting chromosomes 6, 8, 9 and 11 enabled to differentiate the majority of melanomas from nevi but was difficult to automate. Tumor cytogenetic heterogeneity was frequent and could impair FISH testing.

\section{Introduction}

Melanomas are aggressive malignant melanocytic tumors, whereas nevi are benign tumors and far more frequent than melanomas. The main effective therapy of this skin cancer is early and sufficiently large surgical removal of the primary lesion. Histopathological examination is the gold standard to discriminate a malignant melanoma from a benign nevus; however, morphologic criteria are not always consensual enough to provide a good inter- and intra-observer reproducibility. As diagnostic failure may pose medical, psychological and medico-legal problems, there is a requirement for ancillary diagnostic tools to aid pathologists to accurately classify melanocytic tumors (1-9).

A major difference between nevi and melanomas is the presence of numerous and recurrent chromosomal imbalances in melanomas (10-15), which are rare in nevi, with the exception of various particular types, including Harvey rat sarcoma viral oncogene homolog (HRAS) gain (11p15) in certain Spitz nevi, breast cancer 1 associated protein-1 (ubiquitin carboxyterminal hydrolase) (BAP1) loss (3p21.1) in 'BAPomas', neuroblastoma RAS viral oncogene homolog (NRAS) gains (1p13.2) in certain Spitzoid tumors and heterozygote deletion of cyclin-dependent kinase inhibitor $2 A$ (CDKN2A) (9p21) in certain melanocytic dysplastic nevi (10-20). Molecular cytogenetic methods have been proposed to improve the distinction between melanoma and nevi using fluorescence in situ hybridization (FISH) or comparative genomic hybridization (CGH) and CGH array $(21,22)$.

These two methods can be used with formalin-fixed, paraffin-embedded samples from routine dermatopathology practice. FISH requires only a few additional tissue sections compared with immunohistochemical analysis, and provides a morphological-based information concerning the copy number of a limited set of chromosomal loci using an 
epifluorescence microscope, even in small samples. By contrast, CGH on chromosomal preparations and CGH array on DNA microarrays require a larger amount of tissue for DNA extraction and detection of the copy number changes throughout the genome, with potential limitation in case of tumor heterogeneity or small samples, without morphological confrontation.

A so-called 'melanoma FISH test' has been proposed to aid distinguishing between a benign nevus (as a 'FISH-negative lesion') and a malignant melanoma (as a 'FISH-positive lesion') on the basis of a 30 nuclei based-signal count on a single FISH slide concerning chromosome 6 centromere (CEP6), Ras-responsive element-binding protein 1 (RREB1) (6p25), MYB (6q23) and cyclin D1 (CCND1) (11q13) (21,23). In spite of notable performances, additional studies already argue for the requirement of additional FISH analyses with other probes, such as those targeting $C D K N 2 A(9 \mathrm{p} 21)$ and $c-M Y C$ (8q24), to overcome 'melanoma FISH test' false-negative results (24). Melanoma FISH test interpretation requires complex algorithms and certain level of expertise to avoid false-positive results, mainly due to polyploidy in nevi, resulting in copy number gains and FISH so-called 'favor malignant' results $(21,24-29)$. False-positive results could also result from a biased selection of only abnormal nuclei, larger than others at different foci in a lesion, instead of counting all nuclei in a given area, what has been called 'cherry picking' by Busam, who, in a recent review, mentioned the requirement for novel probe sets testing a broader number of chromosomal loci to overcome these problems (26).

In the present study, an alternative test to the classical 'melanoma FISH test' is proposed, which is based on the digital image analysis and detection of intra-chromosomal imbalances in chromosomes 6, 8, 9 and 11 . The present study also attempted to develop a software-assisted FISH count method and a semi-quantitative visual approach. In addition, tumor heterogeneity and its limitation in the detection of chromosomal imbalances is assessed.

\section{Materials and methods}

Case selection. A total of 170 samples from cases analyzed at Department of Pathology, Brest University Hospital (Brest, France) were collected between 2010 and 2012. A first set of 62 cases of non-ambiguous melanoma (47 samples) and nevi (15 samples) with $\geq 2 \mathrm{~mm}$ thickness was selected to be included in tissue microarray (TMA) blocks, and a second set of 108 other tumors (43 primary melanomas, 27 metastases and 38 nevi) was studied on whole-slide sections on the basis of digitalized FISH slides. In this second set of tumors, 12 primary melanomas were analyzed in four different areas for each tumor, and comparisons were made between paired melanoma samples in 10 patients with metastatic melanomas. The pathological data of the two sets of tumors are summarized in Table I.

All samples were included in a registered tumor tissue collection, and the study was conducted in compliance with the Declaration of Helsinki and following approval by the institutional review board of Brest University Hospital (Brest, France; approval no. CPP n DC-2008-214), which included written informed consent obtained from the patients.
Table I. Histological subtypes of the two sets of tumors.

\begin{tabular}{|c|c|c|}
\hline Tumor characteristics & $\begin{array}{c}\text { First set } \\
\text { (62 TMA- } \\
\text { included tumors) }\end{array}$ & $\begin{array}{c}\text { Second set } \\
\text { (108 TMA- } \\
\text { included tumors) }\end{array}$ \\
\hline Primary melanomas & 47 & 43 \\
\hline Superficial spreading & 16 & 16 \\
\hline Nodular & 24 & 8 \\
\hline Acral lentiginous & 3 & 1 \\
\hline Lentigo maligna & 4 & 8 \\
\hline Desmoplastic & 0 & 4 \\
\hline Mucosal & 0 & 2 \\
\hline Unclassifiable & 0 & 3 \\
\hline Nevi & 15 & 38 \\
\hline Junctional & 0 & 8 \\
\hline Dermal & 5 & 11 \\
\hline Compound & 7 & 6 \\
\hline Congenital & 1 & 3 \\
\hline Reed & 2 & 4 \\
\hline Spitz & 0 & 1 \\
\hline Conventional blue & 0 & 3 \\
\hline Cellular blue & 0 & 2 \\
\hline Metastases & 0 & 27 \\
\hline Lymph node & 0 & 13 \\
\hline In-transit metastasis & 0 & 3 \\
\hline Skin & 0 & 9 \\
\hline Lung & 0 & 1 \\
\hline Adrenal gland & 0 & 1 \\
\hline
\end{tabular}

TMA, tissue microarray.

TMA. TMA blocks were built using Tissue-arrayer ${ }^{\circledR}$ (Beecher Instruments Inc., Sun Prairie, WI, USA). For each case, six tumor cores $(0.6-\mathrm{mm}$ diameter) of tumor were transferred from the selected tumor areas to the recipient block. Sections of $5 \mu \mathrm{m}$ were cut on a microtome and transferred to Superfrost ${ }^{\mathrm{TM}}$ glass slides (Thermo Fisher Scientific, Inc., Waltham, MA, USA). The first slides were colored routinely to attest the presence of tumor cells on a spot. For each tumor included, only the most tumor cell-rich and best conserved spot was considered for further FISH analyses. In this manner, the potential impact of likely intra-tumor heterogeneity was minimized by analyzing a single and identical tissue area per TMA-included tumor with the two commercial and bacterial artificial chromosome (BAC) probes FISH assays.

BAC probes. BAC clones corresponding to the DNA sequences included in the regions of interest on chromosomes 6,8 , 9 and 11 were selected, according to the information contained in the UCSC Genome Browser (http://genome.ucsc.edu), and were purchased from the Children's Hospital Oakland Research Institute (Oakland, CA, USA; http://bacpac.chori. org). Two BAC clones were selected for each chromosome, one on the short arm and the other one on the long arm, and labeled with different fluorochromes to enable co-hybridization on a 
Table II. BAC clones used to prepare fluorescence in situ hybridization probes.

\begin{tabular}{llll}
\hline BAC clone & Chromosomal locus & Labeling & Probes sets \\
\hline RP11-61O16 & RREB1 (6p25) & SpectrumRed $^{\mathrm{TM}}$ & Chromosome 6 \\
RP11-323N12 & MYB (6q23.3) & SpectrumGreen $^{\mathrm{TM}}$ & Chromosome 6 \\
RP11-440N18 & $c$-MYC (8q24.1) & SpectrumRed $^{\mathrm{TM}}$ & Chromosome 8 \\
RP11-1084C20 & POTEA (8p11.1) & SpectrumGreen $^{\mathrm{TM}}$ & Chromosome 8 \\
RP11-478M20 & CDKN2A (9p21.3) & SpectrumRed $^{\mathrm{TM}}$ & Chromosome 9 \\
RP11-959B21 & GNA $(9 \mathrm{q} 21.2)$ & SpectrumGreen $^{\mathrm{TM}}$ & Chromosome 9 \\
RP11-156B3 & $C C N D 1(11 \mathrm{q} 13.3)$ & SpectrumRed $^{\mathrm{TM}}$ & Chromosome 11 \\
RP11-1007G14 & HRAS $(11 \mathrm{p} 15.5)$ & SpectrumGreen $^{\mathrm{TM}}$ & Chromosome 11 \\
\hline
\end{tabular}

BAC, bacterial artificial chromosome; RP, Roswell Park; RREB1, Ras-responsive element-binding protein 1; POTEA, prostate, ovary, testisexpressed ankyrin domain family, member A; CDKN2A, cyclin-dependent kinase inhibitor 2A; GNAQ, guanine nucleotide-binding protein $G(q)$ subunit alpha; CCND1, cyclin D1; HRAS, Harvey rat sarcoma viral oncogene homolog.

single slide. BAC extraction was conducted as previously described (30). The extracted DNA was labeled with SpectrumRed $^{\mathrm{TM}}$ or SpectrumGreen ${ }^{\mathrm{TM}}$ fluorochromes (Abbott Molecular, Des Plaines, IL, USA) using Nick Translation kit (Abbott Molecular) following the manufacturer's protocol, and was precipitated into hybridization buffer. Mapping of each BAC was validated by FISH analysis on healthy donor metaphases (Table II).

FISH methods. Five hybridizations were performed in the first set of TMA-included tumors, one with the Vysis Melanoma FISH Probe kit (Abbott Molecular) and four with the four BAC probes pairs. The second set of tumors was studied with BAC probes only. Following deparaffinization and rehydratation, the slides were pretreated with Histology FISH Accessory kit (Dako, Glostrup, Denmark) following the manufacturer's protocol. FISH was performed with a hybridization automaton (HYBrite; Abbott Molecular). Probes were placed on the TMA slide, covered with a glass slide and then sealed with rubber cement (Starkey Chemical Process Co., La Grange, IL, USA). After co-denaturation at $73^{\circ} \mathrm{C}$ during $5 \mathrm{~min}$, the probes and the target DNA were allowed to hybridize at $37^{\circ} \mathrm{C}$ overnight in a humid and dark atmosphere. Then, the excess probes and non-specific hybridizations were eliminated by stringent washing in a bath with $2 \mathrm{X}$ saline sodium citrate and NP-40 at $72^{\circ} \mathrm{C}$. Slides were assembled following air-drying in the darkness and counter-coloration with $14 \mu 1$ 4',6-diamidino-2-phenylindole (DAPI) solution (Vector Laboratories Inc., Burlingame, CA, USA). The first set of TMA-included tumors was read using an epifluorescence microscope at x1,000 magnification (Zeiss AG, Oberkochen, Germany) with DAPI, SpectrumGreen ${ }^{\mathrm{TM}}$, SpectrumRed $^{\mathrm{TM}}$, SpectrumGold ${ }^{\mathrm{TM}}$ and SpectrumAqua ${ }^{\mathrm{TM}}$ filters (Abbott Molecular). The microscope was connected to a chargecoupled device camera and a software (In Situ Ichtyoplankton Imaging System version 5.3; MetaSystems Hard \& Software $\mathrm{GmbH}$, Altlußheim, Germany) for analyzing fluorescent signals, either directly on microscopic examination for the Vysis Melanoma FISH test or on captured images saved as Joint Photographic Experts Group files for BAC probes. For the second set of tumors, FISH slides were scanned using an automated microscope and imaging software (PathScan ${ }^{\circledast}$ FISH; Excilone,
Elancourt, France). Tumor areas were captured and saved as Tagged Image File Format files using the PathScan ${ }^{\circledR}$ Vewer software (Excilone). A field of tumor of $0.06 \mathrm{~mm}^{2}$ (equivalent to $x 40$ magnification) was captured per slide, with the exception of 12 large primary melanomas, where four different areas were captured to search for intra-tumor heterogeneity. FISH analyses, manual and automated, were then performed on the basis of these images, manually and with the ImageJ open source image manipulation tool developed by Mr. Wayne Rasband (National Institutes of Health, Bethesda, MD, USA; http://rsb.info.nih. gov/ij), which is widely used for biomedical image processing. Only tumor cell-rich fields were considered.

Signals counting and FISH interpretation. Previously developed criteria were used to analyze the results of Vysis FISH test. A total of 30 melanocytic nuclei per lesion were directly examined under the microscope. A lesion was considered to be positive if any of the following criteria was met: Gain in 6p25 (RREB1, SpectrumRed ${ }^{\mathrm{TM}}$ ) relative to CEP6 (Spectru$\left.\mathrm{mAqua}^{\mathrm{TM}}\right)>55 \%$, gain in $6 \mathrm{p} 25>29 \%$ ( $>2$ signals/nucleus), loss in 6q23 (MYB, SpectrumGold ${ }^{\mathrm{TM}}$ ) relative to CEP6 $>40 \%$ or gain in 11q13 $\left(C C N D 1\right.$, SpectrumGreen $\left.{ }^{\mathrm{TM}}\right)>38 \%(21,23)$.

To analyze BAC-based hybridization, FISH positivity thresholds were defined on the basis of non-tumor tissue analyses, and any results beyond these thresholds were considered as positive. BAC signals were counted independently of the number of nuclei per picture. Exact signal counts were performed in the first set of TMA-included tumors and as part of the analyses performed in the second set of tumors, using the build-in cell counter tool of ImageJ software to manually point and count every signal within the image. Additional semi-quantitative visual appreciation of green/red signal ratios and automated macro-based signal counts were performed in the second set of tumors and compared with the reference manual exact count.

Statistical analyses. Statistical analyses were performed using MedCalc statistical software version 13.2.2 (MedCalc Software bvba, Ostend, Belgium; http://www.medcalc.org). P<0.05 was considered to indicate a statistically significant difference. Bland-Altman plots were used to evaluate the mean difference 
between manual counts and macro-based automatic method of signal counting in a calibration set of 22 slides. Cohen's kappa coefficient was calculated to evaluate the strength of agreement between the reference manual count and the macro-based automatic method of signal counting in the first set of tumors, and between the reference manual count and the semi-quantitative visual method in the second set of tumors.

\section{Results}

Determination of BAC probes thresholds. Manually counting red and green signals in 15 non-tumor tissues (normal epidermis and lymph nodes) hybridized with the four BAC pairs resulted in a mean green/red signal ratio of 1.0 (ranging from 0.9 to 1.1), with a standard deviation (SD) of 0.05 . The threshold values were defined as the extreme (inferior and superior) values $\pm 2 \times \mathrm{SD}$. As a result, a green/red signal ratio $<0.8$ or $>1.2$ was considered to reflect intra-chromosomal imbalance.

Analyses of the first set of TMA-included tumors with commercial and BAC probes. Within the first set of 62 TMA-included tumors using the commercial FISH probe test, 45/47 (95.7\%) melanomas presented $\geq 1$ positive FISH criterion and 2/47 (4.3\%) were FISH-negative. Among the nevi, 2/15 $(13.3 \%)$ tumors were FISH-positive and 13/15 (86.7\%) were FISH-negative. The 2 FISH-positive nevi presented a polysomy of chromosome 6 in 40 and $30 \%$ of tumor cells, respectively, with gain of $11 \mathrm{q} 13$ signal in $41 \%$ (positive criterion) and $28 \%$ (negative criterion) of tumor cells, respectively, thus reflecting polyploid cells.

With the BAC probes, 47/47 (100.0\%) melanomas presented $\geq 1$ chromosomal imbalance, and only 1 Spitz nevus presented a chromosome 11 imbalance as a $11 \mathrm{p} 15.5$ HRAS amplification, classically described in a subset of Spitz nevi (16). The results of the FISH analyses of the first set of tumors are summarized in Fig. 1.

Analyses of the second set of tumors with BAC probes on digital FISH images. The second set of tumors was analyzed with the four BAC probes pairs by counting manually the green and red signals to obtain green/red signal ratios. Of the $70(7.1 \%)$ malignant lesions (2/43 primary melanomas and 3/27 metastases), 5 did not present an intra-chromosomal imbalance in chromosomes $6,8,9$ or 11 , whereas $\geq 1$ intra-chromosomal imbalance was encountered in the other malignant lesions $(65 / 70,92.9 \%)$. No intra-chromosomal imbalance was observed in any of the 38 nevi. The strength of agreement between the manual count, considered as reference, and the automatic or semi-quantitative visual appreciation methods, was subsequently studied.

A macro-based automatic method of signal counting was developed on the basis of 22 FISH slides of good quality not included in the present lesions set. The developed method exhibited a good correlation in determining a green/red signal ratio in these 22 slides, with a mean difference between the manual count and the automatic method of 0.000 [95\% confidence interval (CI), -0.120 to 0.120$]$. Searching for intra-chromosomal imbalances in the second set of tumors using this automatic method compared with the manual count approach exhibited overall
Table III. Strength of agreement between the manual count and the alternative automatic count and semi-quantitative visual methods.

Kappa-value $(95 \%$ CI)

\begin{tabular}{lcl}
\hline & \multicolumn{2}{c}{ Kappa-value (95\% CI) } \\
\cline { 2 - 3 } Variable & Automatic count & $\begin{array}{c}\text { Semi-quantitative } \\
\text { visual method }\end{array}$ \\
\hline BAC probes & & \\
Chromosome 6 & $0.746(0.627-0.866)$ & $0.726(0.605-0.848)$ \\
Chromosome 8 & $0.402(0.277-0.527)$ & $0.751(0.631-0.871)$ \\
Chromosome 9 & $0.323(0.189-0.458)$ & 1.000 \\
Chromosome 11 & $0.471(0.341-0.601)$ & $0.735(0.609-0.861)$ \\
Image quality & & \\
Good & $0.630(0.536-0.723)$ & $0.846(0.776-0.916)$ \\
Moderate & $0.384(0.277-0.492)$ & $0.846(0.763-0.929)$ \\
Poor & $0.480(0.336-0.625)$ & $0.692(0.557-0.828)$ \\
\hline
\end{tabular}

BAC, bacterial artificial chromosome; CI, confidence interval.

moderate strength of agreement between the two methods (kappa-value, 0.515; 95\% CI, 0.449-0.580). Consideration of the quality of the digital images in the interpretation of the results revealed that the agreement between the manual count and the automatic method was better in good-quality images than in images with moderate-to-poor quality (Table III).

Using the semi-quantitative visual method, the strength of agreement was considered to be good-to-very good (kappa-value, 0.816; 95\% CI, 0.765-0.868). Contrary to the automatic method, the quality of the images did not greatly influence the strength of agreement with the semi-quantitative visual method (Table III). Differences in the results between the manual count and the semi-quantitative visual method did not modify the global positivity/negativity of the BAC FISH assay (no result changed between 'no chromosomal aberration detected' and ' $\geq 1$ chromosomal imbalance detected'), but certain variations consisted of overestimation of gain of red signals with the semi-quantitative method in cases of high polysomy encountered in melanoma samples but not in nevi (Fig. 2). Thus, it appears that the less numerous polysomic cells frequently encountered in nevi did not cause error of signal semi-quantification in the present study.

Intra- and inter-tumor homogeneity/heterogeneity. Manual count of green/red signal ratio was used for each of the chromosomes 6, 8, 9 and 11 to investigate tumor heterogeneity within 12 primary melanomas considering four different areas per tumor, and between paired tumor samples in 10 patients with metastatic melanomas. Intra-tumor heterogeneity was constant within the 12 primary melanomas, as was inter-tumor heterogeneity, which was present in 9 of 10 patients with $\geq 1$ difference between two samples (Fig. 3).

\section{Discussion}

Previous CGH and CGH array analysis of numerous melanocytic tumors revealed that melanomas exhibit multiple 
A

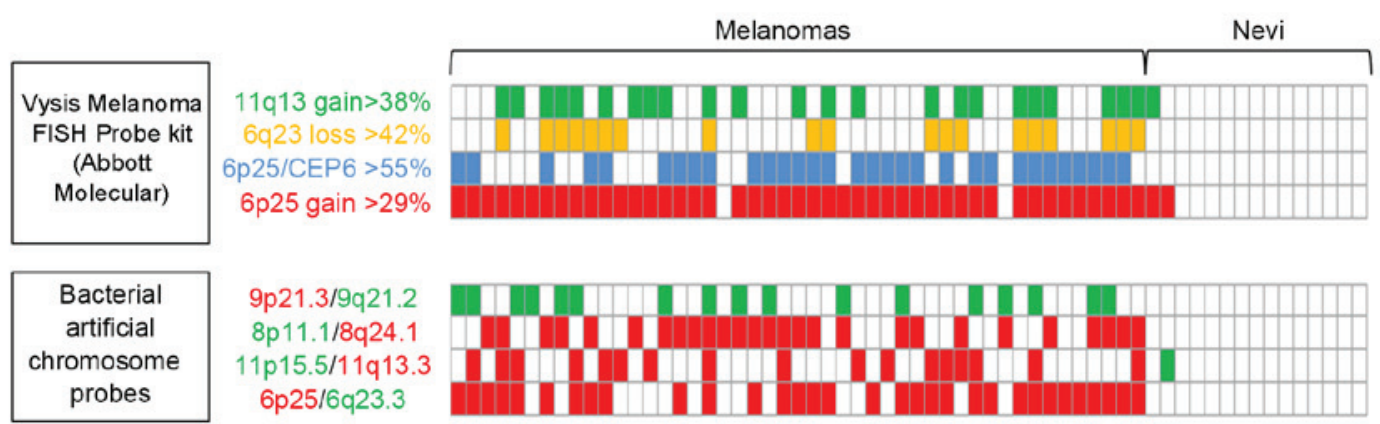

B

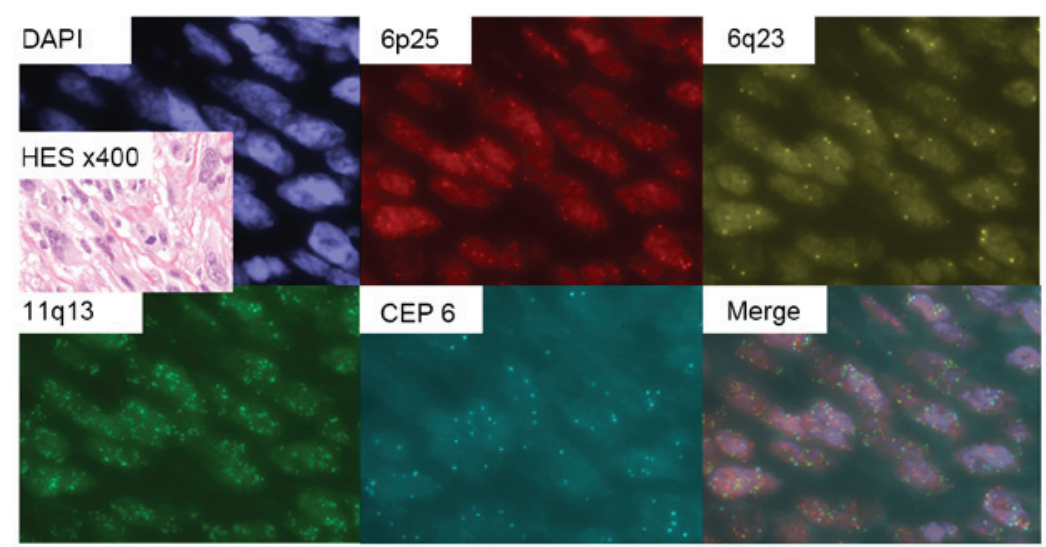

C

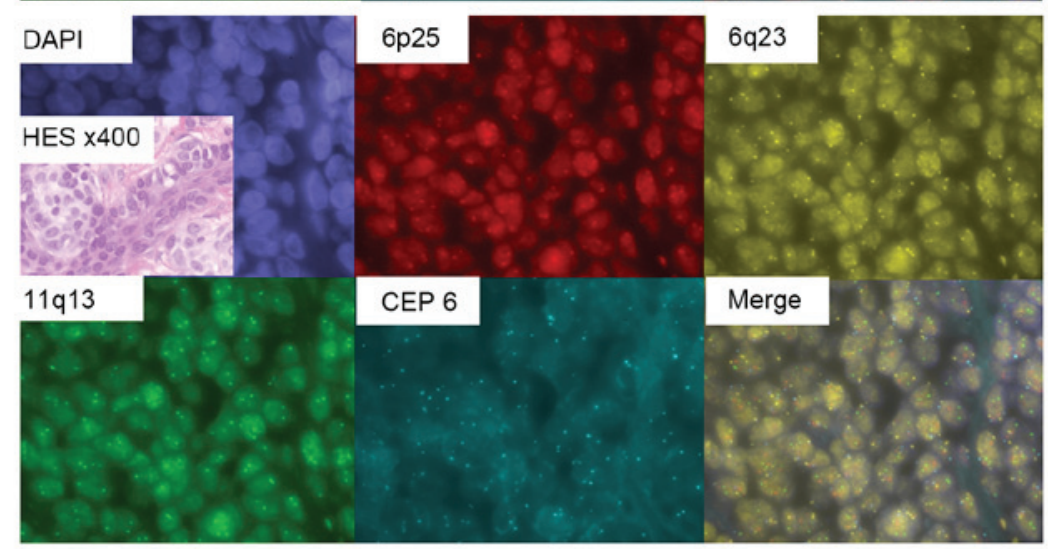

D

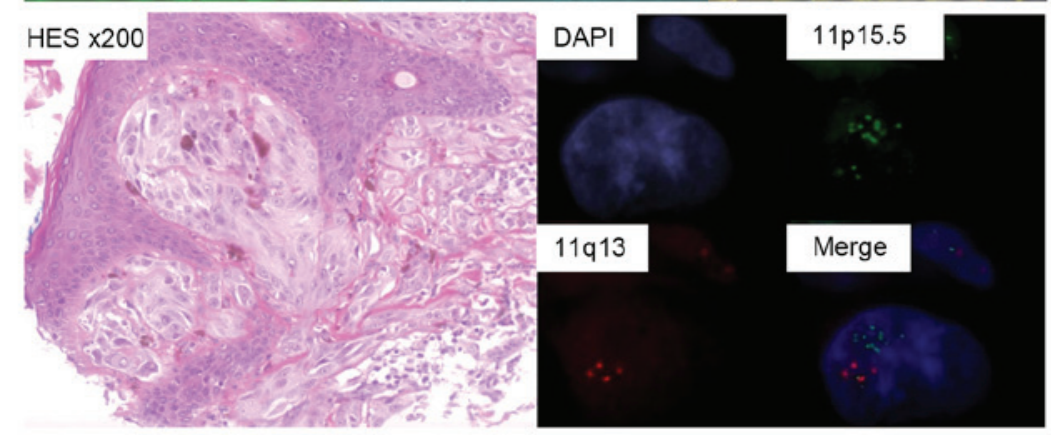

Figure 1. Results of FISH analyses in the tissue microarray-included tumors. (A) Summary of positive (colored cases) and negative (white cases) FISH criteria of the 62 tumors analyzed with commercial and BAC probes. Cases are represented vertically. Using BAC probes, red cases indicated a predominance of red signals, while green cases indicated a predominance of green signals. (B) Example of a FISH-positive melanoma analyzed with the commercial FISH probe, which exhibited a gain of 6p25, a loss of 6q23 and an amplification of 11q13 (split channels and merge; x100 magnification for FISH; x400 magnification for HES tumor histological view). (C) Example of a FISH-negative nevus analyzed with the commercial FISH probe, exhibiting no chromosome 6 or 11 gain or loss (split channels and merge; x100 magnification for FISH; x400 magnification for HES tumor histological view). (D) Focus on a Spitz nevus cell presenting polysomy of chromosome 11 and 11p15.5 (Harvey rat sarcoma viral oncogene homolog) amplification with chromosome 11 BAC probes (split channels and merge; x100 magnification for FISH; x200 magnification for HES tumor histological view). FISH, fluorescence in situ hybridization; BAC, bacterial artificial chromosome; HES, hematoxylin and eosin; DAPI, 4',6-diamidino-2-phenylindole; CEP6, centromere 6.

chromosomal aberrations, whereas only a minority of benign nevi do, particularly certain isolated aberrations in specific loci, such as $C D K N 2 A$ heterozygote deletion, HRAS gain, NRAS gain or $B A P 1$ loss (10-20). Those results enabled the development of targeted FISH based on the most recurrent unbalanced loci observed in melanomas (chromosomes 6 and 11) (21). 
$\mathbf{A}$
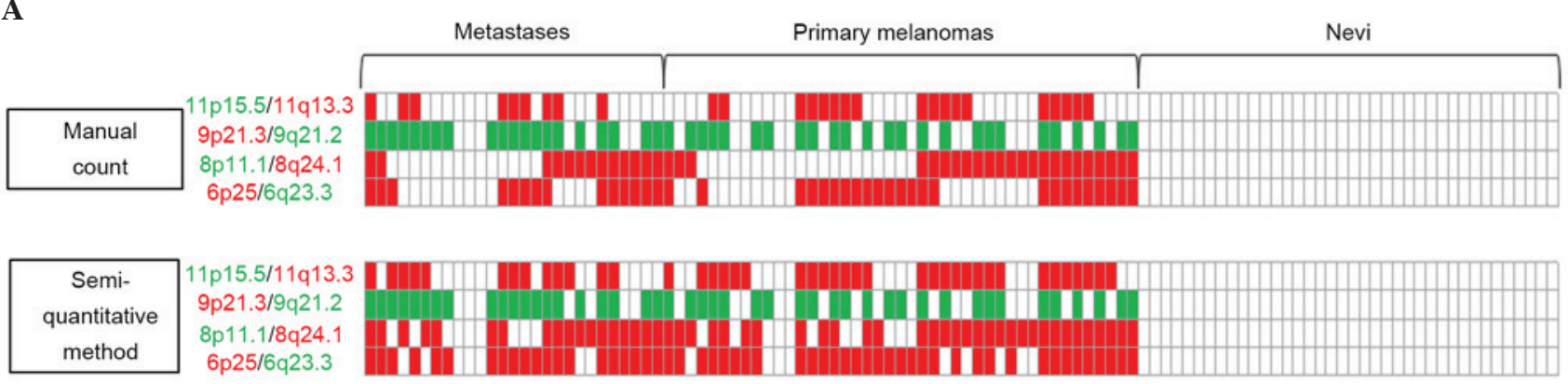

B

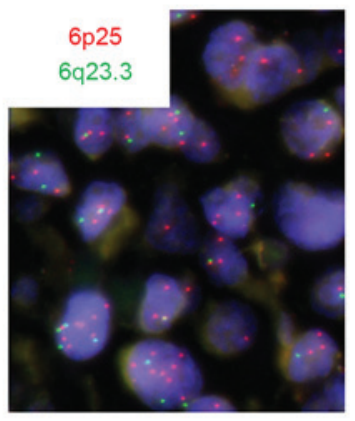

C

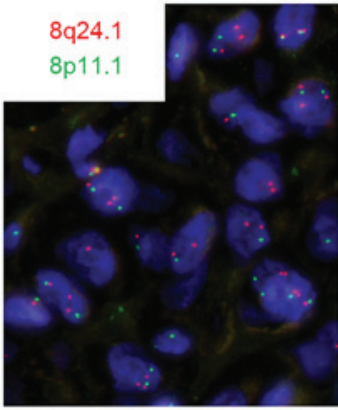

D

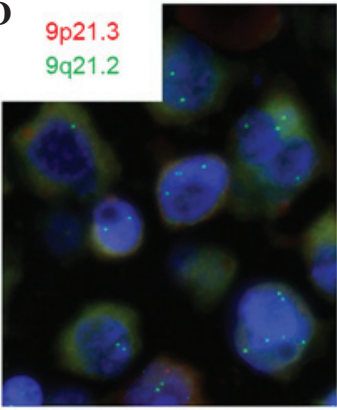

$\mathbf{E}$

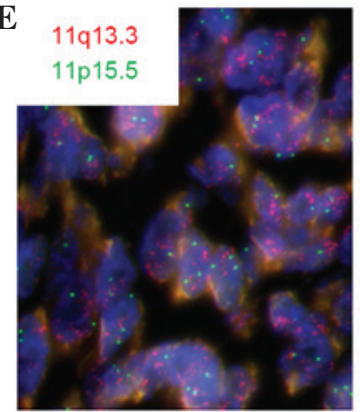

Figure 2. Results of digital FISH images analyses. (A) Summary of positive (colored cases) and negative (white cases) FISH criteria of the 108 tumors with manual count and semi-quantitative method. Cases are represented vertically. Red cases indicate a predominance of red signals, while green cases indicate a predominance of green signals. (B) Example of 6p25 gain in a melanoma (merge, x100 magnification). (C) Example of 8q34.1 gain in a melanoma (merge, x100 magnification). (D) Example of 9p21.3 deletion in a melanoma (merge, x100 magnification). (E) Example of 11q13.3 amplification in a melanoma (merge, x100 magnification). FISH, fluorescence in situ hybridization.

A

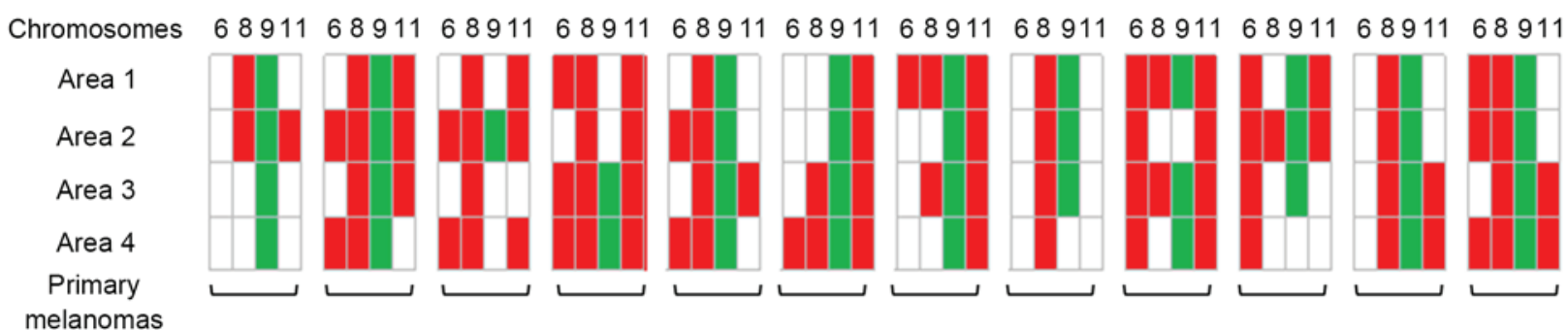

B

Cases
11p15.5/11q13.3
$9 \mathrm{p} 21.3 / 9 \mathrm{q} 21.2$
$8 \mathrm{p} 11.1 / 8 \mathrm{q} 24.1$
6p25/6q23.3
Tumors
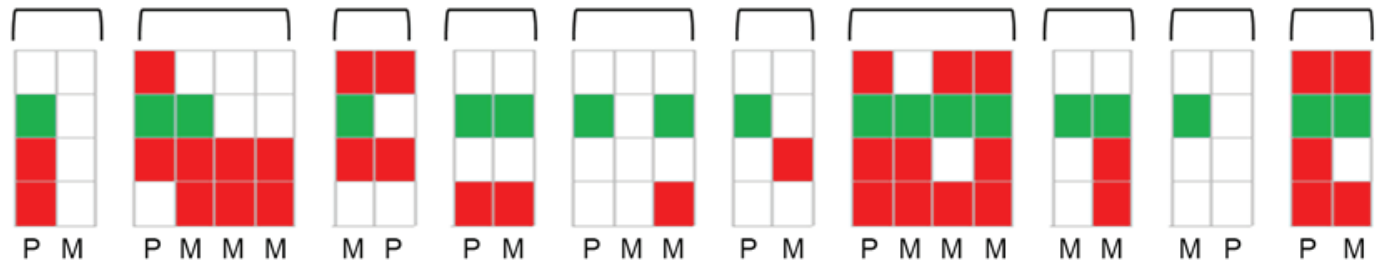

Figure 3. Results of digital FISH image analyses searching for intra-tumor and inter-tumor heterogeneity. Red cases indicate a predominance of red signals, while green cases indicate a predominance of green signals. (A) Summary of positive (colored cases) and negative (white cases) FISH criteria of $12 \mathrm{P}$ with manual count on 4 different tumor areas/tumor. Every tumor presents $\geq 1$ discrepant result between different areas. (B) Summary of positive (colored cases) and negative (white cases) FISH criteria of paired tumor samples of $\mathrm{P}$ and $\mathrm{M}$ in 10 patients with $\mathrm{M}$, as analyzed by manual count (samples are represented vertically). With the exception of 1 patient, multiple differences were observed between paired samples. FISH, fluorescence in situ hybridization; P, primary melanoma; M, metastatic melanoma.

Testing unambiguous nevi demonstrated that nevi may present a mixture of diploid and tetraploid cells, not revealed in $\mathrm{CGH}$ or CGH array studies (26).

Intra-chromosomal imbalances, particularly when they are multiple in a same lesion, appear to be more specific of a malignant melanocytic tumor than polyploidy and whole-chromosome aneuploidy $(12,24,26,29)$. However, the commercial FISH test considers both intra-chromosomal imbalances and whole chromosome gain as malignancy criteria, which leads to a number of false-positive results $(26,29)$. In the present study, 2 nevi were considered as 'malignant' lesions using this commercial FISH test, in spite of definitively clinical and histopathological benign features. Of these 2 lesions, 1 was a tetraploid nevus that was not concluded as a 
genomic-unbalanced lesion with the current eight-probe BAC FISH test, which focuses on intra-chromosomal imbalances. The other lesion was concluded as a specific, but benign, Spitz nevus, displaying an isolated $H R A S$ gain with the current test, whereas the commercial FISH test concluded as a 'malignant' lesion due to tetraploidy appearing as $>2$ signals for loci of chromosomes 6 and 11. Such an isolated HRAS gain is well known as a specific signature of certain Spitz nevi, but is not considered in the commercial FISH test (16). This is unexpected, since the aim of this ancillary cytogenetic analysis is to aid distinguishing between benignity and malignancy in cases of difficult, ambiguous melanocytic lesions that include numerous Spitzoid tumors.

Designing the most efficient FISH probe set to accurately differentiate the majority of nevi from melanomas remains difficult. Attempts were previously made to modify the initial FISH test containing RREBl, MYB, CEP6 and $C C N D 1$ probes by a more discriminatory test employing $R R E B 1, c-M Y C$, $C D K N 2 A$ and $C C N D 1$ probes. This latter test reached a sensitivity of $94 \%$ and a specificity of $98 \%$, which is more efficient that the $75 \%$ sensitivity and $96 \%$ specificity obtained with the former test (24). This improvement is due to a lesser false-positive rate linked to tetraploidy and the fact that the new test takes into account four different chromosomes instead of two. However, FISH test algorithms remain complicated and exposed to numerous technical artifacts. Visual selection of atypical cells solely by the FISH reader is a well-known cause of excessive positivity of the test; this 'cherry-picking' behavior can be overcome using a systematic analysis of every tumor cells in a given microscopic field (26). Another limitation consists in the signal count per nucleus. Artificial signal loss may be due to too-thin tissue sections, whereas nuclei overlapping in too-thick tissue sections may lead to false signal gains. The number of nuclei to be analyzed is also a matter of debate. It is currently recommended to analyze only 30 nuclei, which may be unrepresentative of the whole tumor. However, analyzing more nuclei, loci and probes, in addition to complex diagnosis algorithms, markedly complicates the analysis and makes it 'time-consuming'. Such limitations indicate the requirement for an easier interpretable and more automated test than the ones currently available.

The present eight-probes/four-chromosomes test presents numerous advantages but remains difficult to automate. First, contrary to the CGH approach, FISH analysis enables morphological confrontation, which may be very useful in case of thin or very inflammatory lesions, where tumor cells are diluted in non-tumor epidermal or inflammatory cells, or in case of suspicion of melanoma developed on a pre-existing nevus. This morphological approach also enables the selection of microscopic fields containing a high proportion of apparently unbalanced cells. In addition, working on digital images of these microscopic fields avoids considering only spare atypical cells in signal counting. Taking into account only the absolute number of signals per probe in this given field also overcomes potential artificial losses or gains of signals caused at the single nucleus level by tissue section thickness. An attempt to computerized signal counting in this digital image raises the possibility of rapid analysis of this multiprobe/multichromosome FISH test. However, software-assisted counting requires high-quality image files, and as a consequence, FISH slides with strong signals without background are required. Such a quality has not been reached in every tumor sample included in the present study. This highlights a possible limitation of a fully automated analysis in a routine workflow using samples from different pathology laboratories with potentially different fixation protocols and heterogeneous FISH slides quality. However, besides this fully automated counting, the use of a cell counter such as the built-in one included in ImageJ already avoids errors in signal counting compared with direct microscopic observation counting. A semi-quantitative visual method appears to be also relevant in the present study, but it could be trainingand observer-dependent, thus requiring a learning curve with confrontation of exact signal counts.

Due to these FISH limitations, CGH and CGH array may be considered, which are also partially automated analyses that explore the whole genome of a given tumor sample with an automated fluorescence ratio-based analysis, providing an average profile of chromosomal imbalances of the cells, tumoral or not, contained in the sample used for DNA extraction $(11,22,26)$. However, morphological analysis is not permitted by these analyses. Approximately $30 \%$ of cells presenting a given chromosomal imbalance in the sample is considered as a rational threshold to allow the detection of this imbalance using CGH-based analysis. Therefore, limitations of CGH-based analyses are encountered in samples containing a low ratio of tumor/non-tumor cells, such as inflammatory, regressive or thin tumors. Cytogenetic intra-tumor heterogeneity in melanomas could also impair the CGH-based pangenomic analyses, but, to the best of our knowledge, limited data are available in the literature (22). In the present study, this heterogeneity was obvious within and between melanoma samples. In this manner, we hypothesize that this tumor heterogeneity must be taken into account in the interpretation of CGH and FISH analyses.

To conclude, the present study highlighted that FISH remains an efficient ancillary tool to argue for the malignancy or benignity of a cutaneous melanocytic lesion. It also pointed out limitations in FISH analysis associated with technical pitfalls and tumor biological heterogeneity. Efficient automated image analysis is difficult to calibrate in a FISH workflow, and visual quantitative and semi-quantitative approaches remain more efficient. Besides FISH analysis, CGH-based methods could be technical alternatives, but caution must be observed in their interpretation, taking into account the percentage of tumor cells within the sample and a potential intra-tumor cytogenetic heterogeneity.

\section{Acknowledgements}

The authors would like to thank the 'Fighting Melanoma' association (Paris, France), 'League Against Cancer' (Brest, France) and 'Omnium Group' (Brest, France) for their financial support. The authors would also like to acknowledge, for their technical assistance and valuable advice, Professor Béatrice Vergier, Professor Jean-Philippe Merlio, Dr Elodie Laharanne and Dr Martina Prochazkova-Carlotti (EA2406, Histology and Molecular Pathology of Tumours, University of Bordeaux, Bordeaux, France and Department of Pathology, Centre Hospitalier Universitaire de Bordeaux, Hôpital 
Haut-Lévêque, Pessac, France); Dr Arnaud De la Fouchardière and Dr Daniel Pissaloux (Department of Biopathology, Centre Léon Bérard, Lyon, France); Ms. Nadia Guéganic and Ms. Corine Tous (Department of Cytogenetics and Reproduction, Brest, France); and the pathologists of Brest, Lorient, Quimper, Saint-Brieuc and the Brest Hospital Biobank.

\section{References}

1. Cochran AJ, Bailly C, Cook M, Crotty K, McCarthy S, Mihm M, Mooi W and Sagebiel R: Recommendations for the reporting of tissues removed as part of the surgical treatment of cutaneous melanoma. The association of directors of anatomic and surgical pathology. Am J Clin Pathol 110: 719-722, 1998.

2. Corona R, Mele A, Amini M, De Rosa G, Coppola G, Piccardi P, Fucci M, Pasquini P and Faraggiana T: Interobserver variability on the histopathologic diagnosis of cutaneous melanoma and other pigmented skin lesions. J Clin Oncol 14: 1218-1223, 1996.

3. Farmer ER, Gonin R and Hanna MP: Discordance in the histopathologic diagnosis of melanoma and melanocytic nevi between expert pathologists. Hum Pathol 27: 528-531, 1996.

4. Jackson R: Malignant melanoma: A review of 75 malpractice cases. Int J Dermatol 36: 497-498, 1997.

5. Kempf W, Haeffner AC, Mueller B, Panizzon RG and Burg G: Experts and gold standards in dermatopathology: Qualitative and quantitative analysis of the self-assessment slide seminar at the 17 th colloquium of the international society of dermatopathology. Am J Dermatopathol 20: 478-482, 1998.

6. Lodha S, Saggar S, Celebi JT and Silvers DN: Discordance in the histopathologic diagnosis of difficult melanocytic neoplasms in the clinical setting. J Cutan Pathol 35: 349-352, 2008.

7. Piepkorn MW, Barnhill RL, Cannon-Albright LA, Elder DE, Goldgar DE, Lewis CM, Maize JC, Meyer LJ, Rabkin MS, Sagebiel RW, et al: A multiobserver, population-based analysis of histologic dysplasia in melanocytic nevi. J Am Acad Dermatol 30: 707-714, 1994.

8. Veenhuizen KC, De Wit PE, Mooi WJ, Scheffer E, Verbeek AL and Ruiter DJ: Quality assessment by expert opinion in melanoma pathology: Experience of the pathology panel of the Dutch Melanoma working party. J Pathol 182: 266-272, 1997.

9. Wakely SL, Baxendine-Jones JA, Gallagher PJ, Mullee M and Pickering R: Aberrant diagnoses by individual surgical pathologists. Am J Surg Pathol 22: 77-82, 1998.

10. Balaban G, Herlyn M, Guerry D IV, Bartolo R, Koprowski H, Clark WH and Nowell PC: Cytogenetics of human malignant melanoma and premalignant lesions. Cancer Genet Cytogenet 11: 429-439, 1984.

11. Bastian BC, LeBoit PE, Hamm H, Bröcker EB and Pinkel D: Chromosomal gains and losses in primary cutaneous melanomas detected by comparative genomic hybridization. Cancer Res 58 : 2170-2175, 1998.

12. Bastian BC, Olshen AB, LeBoit PE and Pinkel D: Classifying melanocytic tumors based on DNA copy number changes. Am J Pathol 163: 1765-1770, 2003.

13. Cowan JM, Halaban R and Francke U: Cytogenetic analysis of melanocytes from premalignant nevi and melanomas. J Natl Cancer Inst 80: 1159-1164, 1988.

14. Limon J, Dal Cin P, Sait SN, Karakousis C and Sandberg AA: Chromosome changes in metastatic human melanoma. Cancer Genet Cytogenet 30: 201-211, 1988.
15. Parmiter AH and Nowell PC: The cytogenetics of human malignant melanoma and premalignant lesions. Cancer Treat Res 43: 47-61, 1988.

16. Bastian BC, LeBoit PE and Pinkel D: Mutations and copy number increase of HRAS in Spitz nevi with distinctive histopathological features. Am J Pathol 157: 967-972, 2000.

17. Dubruc E, Balme B, Dijoud F, Disant F, Thomas L, Wang Q, Pissaloux D and de la Fouchardiere A: Mutated and amplified NRAS in a subset of cutaneous melanocytic lesions with dermal spitzoid morphology: Report of two pediatric cases located on the ear. J Cutan Pathol 41: 866-872, 2014.

18. Park WS, Vortmeyer AO, Pack S, Duray PH, Böni R, Guerami AA, Emmert-Buck MR, Liotta LA and Zhuang Z: Allelic deletion at chromosome 9p21(p16) and 17p13(p53) in microdissected sporadic dysplastic nevus. Hum Pathol 29: 127-130, 1998.

19. Sini MC, Manca A, Cossu A, Budroni M, Botti G, Ascierto PA, Cremona F, Muggiano A, D'Atri S, Casula M, et al: Molecular alterations at chromosome 9p21 in melanocytic naevi and melanoma. Br J Dermatol 158: 243-250, 2008.

20. Wiesner T, Murali R, Fried I, Cerroni L, Busam K, Kutzner H and Bastian BC: A distinct subset of atypical Spitz tumors is characterized by BRAF mutation and loss of BAP1 expression. Am J Surg Pathol 36: 818-830, 2012.

21. Gerami P, Jewell SS, Morrison LE, Blondin B, Schulz J, Ruffalo T, Matushek P IV, Legator M, Jacobson K, Dalton SR, et al: Fluorescence in situ hybridization (FISH) as an ancillary diagnostic tool in the diagnosis of melanoma. Am J Surg Pathol 33: 1146-1156, 2009

22. Wang L, Rao M, Fang Y, Hameed M, Viale A, Busam K and Jhanwar SC: A genome-wide high-resolution array-CGH analysis of cutaneous melanoma and comparison of array-CGH to FISH in diagnostic evaluation. J Mol Diagn 15: 581-591, 2013.

23. Vergier B, Prochazkova-Carlotti M, de la Fouchardière A, Cerroni L, Massi D, De Giorgi V, Bailly C, Wesselmann U, Karlseladze A, Avril MF, et al: Fluorescence in situ hybridization, a diagnostic aid in ambiguous melanocytic tumors: European study of 113 cases. Mod Pathol 24: 613-623, 2011.

24. Gerami P, Li G, Pouryazdanparast P, Blondin B, Beilfuss B, Slenk C, Du J, Guitart J, Jewell S and Pestova K: A highly specific and discriminatory FISH assay for distinguishing between benign and malignant melanocytic neoplasms. Am J Surg Pathol 36: 808-817, 2012.

25. Boone SL, Busam KJ, Marghoob AA, Fang Y, Guitart J, Martini M and Gerami P: Two cases of multiple spitz nevi: Correlating clinical, histologic, and fluorescence in situ hybridization findings. Arch Dermatol 147: 227-231, 2011.

26. Busam KJ: Molecular pathology of melanocytic tumors. Semin Diagn Pathol 30: 362-374, 2013.

27. Gerami P and Zembowicz A: Update on fluorescence in situ hybridization in melanoma: State of the art. Arch Pathol Lab Med 135: 830-837, 2011.

28. Isaac AK, Lertsburapa T, Pathria Mundi J, Martini M, Guitart J and Gerami P: Polyploidy in spitz nevi: A not uncommon karyotypic abnormality identifiable by fluorescence in situ hybridization. Am J Dermatopathol 32: 144-148, 2010.

29. Zembowicz A, Yang SE, Kafanas A and Lyle SR: Correlation between histologic assessment and fluorescence in situ hybridization using MelanoSITE in evaluation of histologically ambiguous melanocytic lesions. Arch Pathol Lab Med 136: 1571-1579, 2012.

30. De Braekeleer E, Douet-Guilbert N, Basinko A, Morel F, Le Bris MJ, Férec C and De Braekeleer M: Using bacterial artificial chromosomes in leukemia research: The experience at the university cytogenetics laboratory in Brest, France. J Biomed Biotechnol 2011: 329471, 2011. 